\title{
Влияние интеркалированного водорода на электронное состояние квазисвободного графена на подложке SiC
}

\author{
(C) С.Ю. Давыдов
}

Физико-технический институт им. А.Ф. Иоффре Российской академии наук, 194021 Санкт-Петербург, Россия

Санкт-Петербургский национальный исследовательский университет инорормационных технологий, механики и оптики, 197101 Санкт-Петербург, Россия

E-mail: Sergei_Davydov@mail.ru

(Получена 22 сентября 2016 г. Принята к печати 31 октября 2016 г.)

Для оценки роли интеркалированного водорода в допировании квазисвободного эпитаксиального графена рассмотрены две системы: (i) графен-NH-SiC\{0001\} и (ii) графен-монослой водорода-N $H$-SiC $\{0001\}$, где $\mathrm{N}=4,6$. В случае (i) смещение точки Дирака эпитаксиального графена вызывается электростатическим полем спонтанной поляризации подложки, в случае (ii) - полем двойного электрического слоя, возникающего вследствие адсорбции атомов водорода. Показано, что во втором случае по сравнению с первым концентрация дырок в графене увеличивается, а концентрация электронов уменьшается, что соответствует имеющимся экспериментальным данным.

DOI: $10.21883 /$ FTP.2017.05.44427.8410

\section{1. Введение}

Исследование влияния подложки на свойства находящейся на ее поверхности пленки графена представляет несомненный интерес. Впервые вопрос о роли спонтанной поляризации $P_{\mathrm{sp}}$ некубических $\mathrm{SiC}$-подложек в допировании сформированного на них однослойного эпитаксиального графена $(Э Г)^{1}$ был рассмотрен в работах $[1,2]$. В работе [1] идея об определяющей роли $P_{\mathrm{sp}}$ была выдвинута для объяснения различий между сильно связанным с $6 H$-SiC(0001)-подложкой ЭГ, обладающим $n$-типом проводимости [3], и демонстрирующим $p$-тип проводимости квазисвободным ЭГ, полученным путем интеркаляции атомарного водорода [4]. В дальнейшем плодотворность этой идеи была обоснована в работе [5].

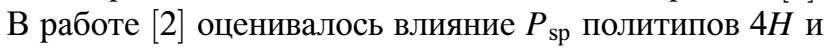
$6 H$ карбида кремния на электронную структуру углеродного буферного слоя, сильно связанного с подложкой, и квазисвободного ЭГ. При этом рассматривались как $\mathrm{Si}-$, так и С-грани подложки, но пассивация этих граней водородом не учитывалась. Было показано, в частности, что в случае, когда точка Дирака свободного (невозмущенного подложкой) графена совпадает с уровнем Ферми подложки, слой графена, сформированный на $\mathrm{SiC}(0001)$, т.е. на Si-грани, заряжается отрицательно, a в случае $\mathrm{SiC}(000 \overline{1})$, т. е. на С-грани, положительно.

Численный расчет (в рамках формализма функционала плотности) для квазисвободного графена на пассивированных водородом подложках $\mathrm{N} H-\mathrm{SiC}(0001)(\mathrm{N}=4,6)$ был выполнен в работах [6,7]. При этом вычислялись соответствующие значения $P_{\mathrm{sp}}$ и диэлектрические воспри-

\footnotetext{
1 Здесь и в дальнейшем под ЭГ мы будем понимать как сильно связанный с подложкой графеновый монослой (в том числе и буферный слой), так и квазисвободный графен. Отметим, что в некоторых работах под ЭГ понимается только графен, сильно связанный с подложкой.
}

имчивости политипов карбида кремния, на основании чего и определялись характер и уровень допирования ЭГ.

Здесь мы рассмотрим электронное состояние однослойного ЭГ на поверхностях (0001) и (0001) 4H- и $6 H$-SiC как в отсутствие, так и при наличии интеркалированного водорода. Основная цель работы состоит в том, чтобы выяснить природу влияния пассивированных водородом оборванных связей $\mathrm{SiC}$-подложки на зарядовое состояние ЭГ. Для этого мы рассмотрим две модели квазисвободного ЭГ: при наличии и в отсутствие монослоя водорода на $\mathrm{SiC}$-подложке.

\section{2. Оценки параметров задачи}

Начнем с простых оценок энергетических характеристик бинарных связей атомов $\mathrm{Si}, \mathrm{C}$ и $\mathrm{H}$, воспользовавшись (здесь и в дальнейшем) методом связывающих орбиталей Харрисона [8-10]. Как показано в работе [2], энергии $s p^{3}$-орбиталей атомов $\mathrm{C}$ и $\mathrm{Si}$ карбида кремния равны соответственно $\varepsilon_{\mathrm{sub}}(C)=-13.15$ эВ и $\varepsilon_{\text {sub }}(\mathrm{Si})=-9.39$ эВ. При этом электроны переходят с атомов кремния на атомы углерода. Результирующий диполь элементарной ячейки, определяющий вектор $\mathbf{P}_{\mathrm{sp}}$, направлен от отрицательного иона к положительному иону, т.е. навстречу $c$-оси $[2,11,12]$. Напряженность наведенного $P_{\mathrm{sp}}$ электрического поля на границе образец/вакуум равна $\mathbf{F}_{\mathrm{sp}}=\mathbf{P}_{\mathrm{sp}} / \varepsilon_{0}$, где $\varepsilon_{0}-$ диэлектрическая постоянная, будет положительной для Si-грани и отрицательной для C-грани [2]. Следовательно, поле $\mathrm{Si}$-грани сдвигает все уровни ЭГ вниз по шкале энергии, а поле С-грани - вверх. В первом случае графен приобретает дополнительные электроны и заряжается отрицательно, во втором случае теряет электроны (приобретает дырки) и заряжается положительно. Величина энергетического сдвига уровней равна $u=-e F_{\text {sp }} l$, где $l-$ расстояние 
от поверхности подложки до листа графена. При этом точка Дирака свободного однослойного графена $\varepsilon_{\mathrm{D} 0}$ смещается в положение $\varepsilon_{\mathrm{D}}=\varepsilon_{\mathrm{D} 0}+u$.

Рассмотрим $\sigma$-связи $\mathrm{C}-\mathrm{H}$ и $\mathrm{Si}-\mathrm{H}$, образованные $s p^{3}$-орбиталями атомов $\mathrm{C}$ и $\mathrm{Si}$ и $s$-орбиталью атома водорода, энергию которой мы примем равной потенциалу ионизации $I=-13.60$ эВ [13]. Ясно, что в обоих случаях электроны переходят на атомы Н. Логично предположить, что при адсорбции атомов водорода на Си Si-гранях карбида кремния имеет место аналогичный переход заряда. Возникающее при этом электростатическое поле сдвигает точку Дирака вверх, что ведет к переходу дырок на ЭГ.

Перейдем теперь к оценкам зонной диаграммы системы карбид кремния-графен. Принимая за нуль энергии центры запрещенной зоны карбида кремния, получим для энергии точки Дирака свободного графена значение $\varepsilon_{\mathrm{D} 0}=\chi+E_{g} / 2-\phi_{g}$, где $E_{g}$ и $\chi-$ соответственно ширина запрещенной зоны и электронное сродство политипа карбида кремния, $\phi_{g}$ - работа выхода свободного графена. Согласно данным работы [14], для $4 H$-политипа $E_{g}=3.23$ и $\chi=3.17$ эВ, для $6 H$-политипа $E_{g}=3.00$ и $\chi=3.45$ эВ. По данным работы [15] имеем $\phi_{g}=5.11$ эВ (именно это значение $\phi_{g}$ использовалось в работе [2]), тогда как в работе [16] приводится значение $\phi_{g}=4.26$ эВ. Такой разброс значений $\phi_{g}$ приводит, вообще говоря, к значимым различиям. Так, в первом случае энергия точки Дирака $\varepsilon_{\mathrm{D} 0}=-0.33$ эВ для $4 H$ и $\varepsilon_{\mathrm{D} 0}=-0.16$ эВ для $6 H$, тогда как во втором имеем $\varepsilon_{\mathrm{D} 0}=0.53$ эВ для $4 H$ и $\varepsilon_{\mathrm{D} 0}=0.69$ эВ для $6 H$ политипов. Для дальнейших оценок примем наиболее часто используемое значение $\phi_{g}=4.50$ эВ. Тогда для политипа $4 H$ получим $\varepsilon_{\mathrm{D} 0}=0.29$ эВ, а для $6 H$ имеем $\varepsilon_{\mathrm{D} 0}=0.45$ эВ.

Энергию одноэлектронного уровня адатома водорода относительно вакуума принимаем равной $\varepsilon_{a 0}=-A-e^{2} / 4 d \quad[17], \quad$ где сродство к электрону $A=0.75$ эВ [13], а длина связи водород-подложка $d=1.88 \AA$, что соответствует расстоянию между ближайшими соседями в карбиде кремния. Тогда имеем $\varepsilon_{a 0}=-2.66$ эВ. Относительно центров запрещенных зон политипов карбида кремния энергия уровня адатома водорода равна $\varepsilon_{a}=\varepsilon_{a 0}+\chi+E_{g} / 2$, откуда $\varepsilon_{a}=2.12$ эВ для $4 H-\mathrm{SiC}$ и $\varepsilon_{a}=2.29$ эВ для $6 H-\mathrm{SiC}$. Матричный элемент $\sigma$-связи $s$-орбитали атома водорода с $s p^{3}$-орбиталями атомов С и $\mathrm{Si}$ подложки равен $V=4.07$ эВ (см. соответствующие формулы в работе [18]). При этом считаем, что каждый атом водорода находится над атомом подложки и связан непосредственно только с этим атомом (см., например, [4]).

\section{3. Зарядовое состояние эпитаксиального графена}

\section{1. Система графен-SiC $\{0001\}$}

Для начала рассмотрим квазисвободный ЭГ на Си Si-гранях карбида кремния в отсутствие интеркали- рованного слоя. ${ }^{2}$ Как было показано выше, без учета влияния спонтанной поляризации энергии точки Дирака равны $\varepsilon_{\mathrm{D} 0}=0.29$ эВ для $4 H$ и $\varepsilon_{\mathrm{D} 0}=0.45$ эВ для $6 H$. Сдвиг точки Дирака в поле спонтанной поляризации равен $u= \pm e\left|P_{\mathrm{sp}}\right| l / \varepsilon_{0}[2]$, где верхний знак относится к С-грани, нижний - к Si-грани. Имеющиеся в литературе расчетные значения $P_{\text {sp }}$ существенно различаются [11], а экспериментальные данные вообще отсутствуют. Здесь мы воспользуемся результатами наших оценок $\left|P_{\mathrm{sp}}\right|=1.13 \cdot 10^{-2}$ Кл/ $\mathrm{M}^{2}$ для $4 H$ и $0.75 \cdot 10^{-2}$ Кл $/ \mathrm{M}^{2}$ для $6 H$, основанных, как и выкладки настоящей работы, на методе связывающих орбиталей Харрисона [8-10]. Следует, однако, отметить, что принятые нами значения $P_{\text {sp }}$ заметно ниже результатов численных расчетов (см. работы [6] и [11]).

Принимая для квазисвободного графена расстояние графен-подложка $l=3 \AA$ (т.е. порядка межслойного расстояния в графите), имеем $|u|=0.38$ эВ для $4 H$-политипа и 0.25 эВ для $6 H$-политипа. Тогда для С-грани получаем $\varepsilon_{\mathrm{D}}=0.67$ эВ для $4 H$ и 0.70 эВ для $6 H$; для $\mathrm{Si}-г$ рани имеем $\varepsilon_{\mathrm{D}}=-0.09$ эВ для $4 H$ и 0.20 эВ для $6 H$.

Пусть теперь между подложкой и квазисвободным графеном имеет место туннелирование электронов, но матричный элемент взаимодействия квазисвободного графена с подложкой мал настолько, что гибридизационным сдвигом точки Дирака можно пренебречь (см. подробнее в работе [17]). Тогда при нулевой температуре графен заряжается отрицательно, если уровень Ферми подложки $\varepsilon_{\mathrm{F}}>\varepsilon_{\mathrm{D}}$, и положительно, если $\varepsilon_{\mathrm{F}}<\varepsilon_{\mathrm{D}}$. Концентрацию носителей в допированном графене легко оценить следующим образом. В пределе слабой связи графен-подложка плотность состояний ЭГ можно аппроксимировать плотностью состояний свободного графена, равной $\rho_{g}=(2 / \pi \sqrt{3})\left(|\omega| / t^{2}\right)$, где $\omega-$ текущая энергия, $t \approx 2.7$ эВ - энергия перехода между ближайшими соседями в графене $[17,19]$. Тогда заряд атома графена есть $Z_{g}= \pm\left(\varepsilon_{\mathrm{D}}-\varepsilon_{\mathrm{F}}\right)^{2} / \pi \sqrt{3} t^{2}$, где верхний (нижний) знак отвечает неравенству $\varepsilon_{\mathrm{D}}>\varepsilon_{\mathrm{F}}\left(\varepsilon_{\mathrm{D}}<\varepsilon_{\mathrm{F}}\right)$. Величина концентрации носителей заряда в ЭГ определяется как

$$
c=\frac{1}{\pi \sqrt{3} S_{g}} \frac{\left(\varepsilon_{\mathrm{D}}-\varepsilon_{\mathrm{F}}\right)^{2}}{t^{2}},
$$

где $c$ равно концентрации электронов $n$ при $\varepsilon_{\mathrm{D}}<\varepsilon_{\mathrm{F}}$ и концентрации дырок $p$ при $\varepsilon_{\mathrm{D}}>\varepsilon_{\mathrm{F}}, S_{g}=3 \sqrt{3} d_{g}^{2} / 4-$ площадь, приходящаяся на один атом графена, $d_{g}=1.42 \AA-$ расстояние между ближайшими соседями в графене.

На рисунке представлены результаты расчета концентраций носителей заряда в квазисвободном ЭГ в функции от положения уровня Ферми внутри запрещенных зон $4 H-\mathrm{SiC}(a)$ и $6 H-\mathrm{SiC}(b)$. При смещении уровня Ферми $\varepsilon_{\mathrm{F}}$ от потолка валентной зоны $E_{V}=-E_{g} / 2$ к дну зоны проводимости $E_{C}=-E_{g} / 2$ характер проводи-

\footnotetext{
2 Это чисто модельная система, где наличие буферного слоя игнорируется.
} 
мости ЭГ меняется в точке Дирака $\varepsilon_{\mathrm{D}}$ с дырочной на электронную. Отметим, что зависимость $c$ от $\left(E_{\mathrm{F}}-\varepsilon_{\mathrm{D}}\right)$, аналогичная представленным на рисунке, была получена в работе [6] путем численных расчетов (см. Fig. 2, $b$ в работе [6]).
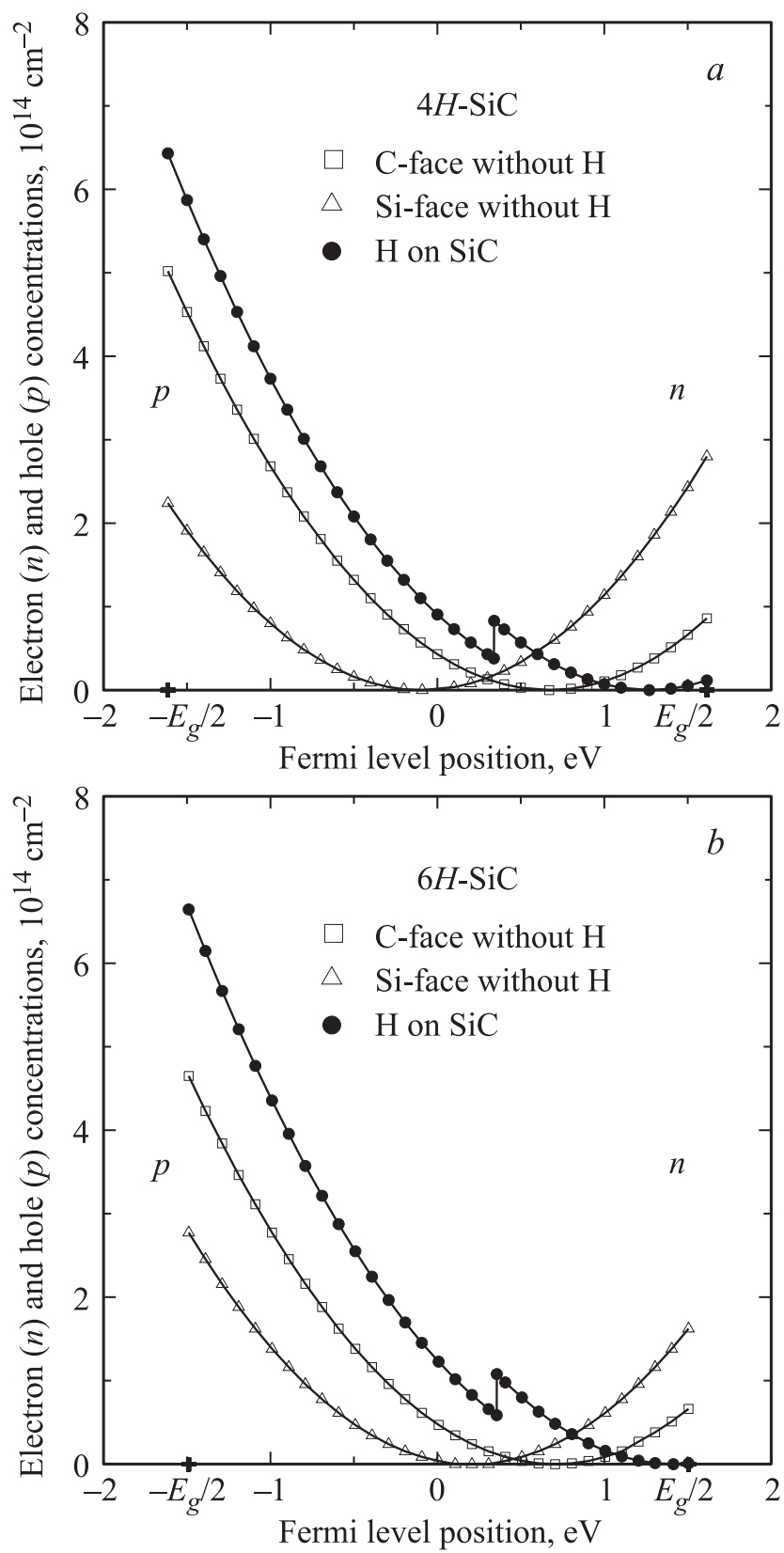

Зависимости концентраций носителей заряда в квазисвободном графене (электронов $n$ и дырок $p$ в ед. $10^{14} \mathrm{~cm}^{-2}$ ) от положения уровня Ферми ( $\varepsilon_{\mathrm{F}}$ в эВ) внутри запрещенных зон $\left(-E_{g} / 2\right.$, $\left.E_{g} / 2\right)$ политипов $4 H-\mathrm{SiC}(a)$ и $6 H-\mathrm{SiC}(b)$. Светлыми символа-

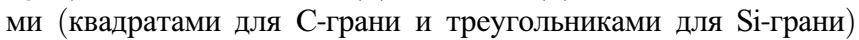
обозначены зависимости в отсутствие водорода, темными кружками - при наличии водорода. Концентрации носителей обращаются в нуль в соответствующих точках Дирака $\varepsilon_{\mathrm{D}}$ и испытывают скачок, когда $\varepsilon_{\mathrm{F}}$ совпадает с энергией локального состояния $\omega_{l}$, наводимого адатомом водорода.
Система графен-водород-SiC\{0001\}: значения зонных $n_{v}$ и локальных $n_{l}$ вкладов в число заполнения изолированного адатома водорода; энергии локального уровня $\omega_{l}$ (в эВ) изолированного адатома водорода относительно центра запрещенной зоны

\begin{tabular}{c|c|c}
\hline Подложка & $4 H-\mathrm{SiC}$ & $6 H-\mathrm{SiC}$ \\
\hline$n_{v}$ & 0.36 & 0.36 \\
$\omega_{l}$ & 0.34 & 0.35 \\
$n_{l}$ & 0.16 & 0.15
\end{tabular}

\section{2. Система графен-водород-SiC $\{0001\}$}

Для оценки перехода заряда в системе графенводород-SiC-субстрат будем следовать работе [20], где вместо водорода рассматривалась интеркаляция атомов $\mathrm{Cu}, \mathrm{Ag}$ и $\mathrm{Au}$. Начнем с рассмотрения невзаимодействующих атомов водорода, адсорбированных на $\mathrm{SiC}$-подложке. Воспользовавшись выражениями (6)-(9) работы [20], получим приведенные в таблице значения зонных $n_{v}$ и локальных $n_{l}$ вкладов в число заполнения адатома водорода $n_{a}=n_{v}+\vartheta\left(\varepsilon_{\mathrm{F}}-\omega_{l}\right) n_{l}$, где $\omega_{l}-$ положение локального уровня, $\vartheta(.)-$. функция Хэвисайда.

Согласно данным работы [21], напряженность электростатического поля дипольного слоя, образованного заряженными адатомами и их изображениями в подложке, в плоскости расположения адатомов $z=0$ равна $F_{a}(0)=-2 e A^{\prime} Z_{a} d / S_{a}^{3 / 2}$, где $S_{a}=3 \sqrt{3} a^{2} / 4-$ площадь, приходящаяся на один адатом водорода, $a \approx 3.08 \AA-$ постоянная решетки для $4 H$ - и $6 H$-политипов [22], $A^{\prime} \sim 10-$ множитель (типа постоянной Маделунга), слабо зависящий от структуры адсорбированного слоя. Учтем, что, в отличие от постоянного во внешнем пространстве электростатического поля спонтанной поляризации $F_{\mathrm{sp}}$, поле двойного электрического слоя резко совпадает с расстоянием $z$ вдоль внешней нормали к поверхности подложки. ${ }^{3}$ Пусть, как и в работе [20], $F_{a}(z)=F_{a}(0) \exp (-\alpha z)$, где $\alpha-$ обратная длина затухания. Тогда сдвиг точки Дирака есть $u=-e F_{a}(l) l$, или

$$
u=-2 e^{2} A^{\prime} Z_{a} d l \exp (-\alpha l) S_{a}^{-3 / 2} .
$$

Предположив $\alpha=1 \AA^{-1}$ и $l=3 \AA$, получим для рассматриваемой нами задачи $u \approx n_{a} d$, где $u$ измеряется в эВ, $d-\mathrm{B} \AA$.

Отметим, что в энергетическом интервале $\left(-E_{g} / 2, \omega_{l}\right)$ входящий в выражение (2) заряд адатома $Z_{c}=-n_{v}$, в интервале $\left(\omega_{l}, E_{g} / 2\right)$ имеем $Z_{a}=-\left(n_{v}+n_{l}\right)$. Тогда для $4 H$-политипа получаем: в интервале от -1.615 до 0.34 эВ сдвиг $u=0.68$ эВ и $\varepsilon_{\mathrm{D}}=0.97$ эВ; в интервале от 0.34 до 1.615 эВ сдвиг $u=0.98$ эВ и $\varepsilon_{\mathrm{D}}=1.27$ эВ. Для $6 H$-политипа имеем: в интервале от -1.5 до 0.35 эВ сдвиг $u=0.68$ эВ и $\varepsilon_{\mathrm{D}}=1.13$ эВ; в интервале от 0.35 до 1.5 эВ сдвиг $u=0.96$ эВ и $\varepsilon_{\mathrm{D}}=1.41$ эВ.

\footnotetext{
${ }^{3}$ Напомним, что в случае классического конденсатора с непрерывным распределением зарядов по обкладкам, электрическое поле во внешнем пространстве равно нулю.
} 
Из рисунка следует, что электростатическое поле, порождаемое монослоем водорода, сдвигает точку Дирака по направлению к верхней границе запрещенной зоны, так что энергетическая область, отвечающая $p$-типу проводимости, расширяется. При этом концентрация дырок возрастает, а концентрация электронов понижается.

Если разность $\varepsilon_{\mathrm{D}}-\varepsilon_{\mathrm{F}}$ (в табл. 1 работы [6] эта разность обозначена как $\Delta D P$ ) меняется от 0.1 до 0.3 эВ, то, согласно нашим расчетам, для $6 H$-политипа концентрация дырок $p$ возрастает с $3.8 \cdot 10^{12}$ до $15.4 \cdot 10^{12} \mathrm{~cm}^{-2}$. Эти оценки отлично согласуются с концентрациями, приведенными в табл. 1 работы [6].

\section{4. Обсуждение результатов}

В предыдущем разделе мы не учитывали взаимодействие адатомов, ведущее, как известно [17], к их деполяризации, т. е. уменьшению величины заряда, в результате чего величина сдвига точки Дирака $|u|$ уменьшается. Введем диполь-дипольное отталкивание адатомов. Соответствующая константа взаимодействия, необходимая для оценки $\bar{n}_{v}$ и $\bar{n}_{l}$ (здесь и далее черта над символом относится к монослою адатомов), равна в нашем случае $\xi=2 e^{2} A^{\prime} d^{2} / S_{a}^{3 / 2} \approx 23.5$ эВ [17,20,23]. Под влиянием этого взаимодействия энергия квазиуровня изолированного адатома $\varepsilon_{a}$ переходит в $\bar{\varepsilon}_{a}=\varepsilon_{a}+\xi \bar{n}_{a}$. Заменяя в формулах (6)-(9) $\varepsilon_{a}$ на $\bar{\varepsilon}_{a}$, самосогласованным образом найдем зонные $\bar{n}_{v}$ и локальные $\bar{n}_{l}$ вклады в $\bar{n}_{a}$.

Рассмотрим, для простоты, только $i$ - и $p$-типы $\mathrm{SiC}$-подложек, когда уровень Ферми находится в нижней половине запрещенной зоны. Так как энергия локального уровня $\bar{\omega}_{l}>0, \bar{n}_{a}=\bar{n}_{v}$, так что для самосогласованного расчета достаточно воспользоваться только выражением (9) из работы [20]. Расчет дает $\bar{n}_{a}=0.26$ для политипов $4 H$ и $6 H$, откуда получаем $\bar{u}=0.49$ эВ, так что $\varepsilon_{\mathrm{D}}=0.78$ эВ для $4 H-\mathrm{SiC}$ и 0.94 эВ для $6 H-\mathrm{SiC}$. Это в полтора раза меньше соответствующих значений $\varepsilon_{\mathrm{D}}=1.13$ и 1.41 эВ для изолированных адатомов, но больше, чем в отсутствие водорода, где максимальное значение $\varepsilon_{\mathrm{D}}=0.70$ эВ имеет место для С-грани $6 H$-политипа. Следовательно, вывод о том, что интеркаляция водорода приводит к расширению дырочной области квазисвободного ЭГ, остается в силе.

\section{5. Заключение}

Итак, мы рассмотрели два варианта допирования квазисвободного графена в отсутствие и при наличии интеркалированного водорода. В первом случае смещение точки Дирака вызывается электростатическим полем спонтанной поляризации некубической $\mathrm{SiC}$-подложки, во втором - полем двойного электрического слоя, возникающего за счет адатомов водорода. Показано, что во втором случае концентрация дырок в графене увеличивается, а концентрация электронов уменьшается. При этом говорить о роли спонтанной поляризации подложки при наличии водорода уже не следует.
Нужно иметь в виду, что в отсутствие непротиворечивых экспериментальных данных приходится использовать чисто теоретические значения исходных параметров задачи (таких, например, как $\left.\phi_{g}, \chi, P_{\mathrm{sp}}, l, \alpha\right){ }^{4}$ Поэтому приведенные в работе результаты следует рассматривать как оценочные, но достаточно удовлетворительно коррелирующие с результатами численных расчетов [6,7].

\section{Список литературы}

[1] J. Ristein, S. Mammadov, Th. Seyller. Phys. Rev. Lett., 108, 246104 (2012).

[2] С.Ю. Давыдов. ФТП, 46, 1209 (2012).

[3] P.N. First, W.A. de Heer, T. Seyller, C. Berger, J.A. Stroscio, J.-S. Moon. MRS Bulletin, 35, 296 (2010).

[4] F. Speck, M. Ostler, J. Röhrl, J. Jobst, D. Waldmann, M. Hundhausen, L. Ley, H.B. Weber, T. Seyller. Appl. Phys. Lett., 99, 122106 (2011).

[5] S. Mammadov, J. Ristein, R.J. Koch, M. Ostler, C. Raidel, M. Wanke, R. Vasiliauskas, R. Yakimova, Th. Seyller. 2D Materials, 1, 035003 (2014).

[6] J. Slawinska, H. Aramberri, M.C. Munoz, J.I. Cerda. Carbon, 93, 88 (2015).

[7] J. Slawinska, H. Aramberri, J.I. Cerda. arXiv:1501.06486.

[8] W.A. Harrison. Phys. Rev. B, 27, 3592 (1983).

[9] W.A. Harrison. Phys. Rev. B, 31, 2121 (1985).

[10] С.Ю. Давыдов, О.В. Посредник. Метод связывающих орбиталей в теории полупроводников. Учеб. пособие. (СПб.: Изд-во СПбГЭТУ „ЛЭТИ“, 2007). 96 с. Электронный адрес: twirpx.com/file/1014608/

[11] С.Ю. Давыдов, А.В. Трошин. ФТТ, 49, 723 (2007).

[12] С.Ю. Давыдов. ФТТ, 51, 1161 (2009).

[13] Физические величины. Справочник, под ред. И.С. Григорьева, Е.3. Мейлихова. (М., Энергоатомиздат, 1991).

[14] С.Ю. Давыдов. ФТТ, 41, 718 (2007).

[15] A. Mattausch, O. Pankratov. Phys. Rev. Lett., 99, 076802 (2007).

[16] K.T. Chan, L.B. Neaton, M.L. Cohen. Phys. Rev. B, 77, 235430 (2008).

[17] С.Ю. Давыдов. Теория адсорбиии: метод модельных гамильтонианов (СПб., Изд-во СПбГЭТУ „ЛЭТИ““, 2013). Электронный адрес: twirpx.com/file/1596114/

[18] С.Ю. Давыдов, Г.И. Сабирова. Письма ЖТФ, 37 (11), 51 (2011).

[19] A.H. Castro Neto, F. Guinea, N.M.R. Peres, K.S. Novoselov, A.K. Geim. Rev. Mod. Phys., 81, 109 (2009).

[20] С.Ю. Давыдов. ФТТ, 56, 406 (2014).

[21] J.P. Muscat, D.M. Newns. J. Phys. C: Sol. St. Phys., 7, 2630 (1974).

[22] В.И. Гавриленко, А.М. Грехов, Д.В. Корбутяк, В.Г. Литовченко. Оптические свойства полупроводников. Справочник. (Киев, Наук. думка, 1987).

[23] С.Ю. Давыдов, А.А. Лебедев, О.В. Посредник. Элементарное введение в теорию наносистем (СПб., Изд-во „Лань“, 2014).

Редактор А.Н. Смирнов

\footnotetext{
${ }^{4}$ Подчеркнем, однако, что ни один параметр не использовался нами как подгоночный.
} 


\title{
Effect of intercalated hydrogen \\ on the electronic state \\ of the quasi-free-standing graphene \\ on $\mathrm{SiC}$ substrate
}

\section{S.Yu. Davydov}

loffe Institute,

194021 St. Petersburg, Russia

St. Petersburg National Research University of Information Technologies, Mechanics and Optics, 197101 St. Petersburg, Russia

\begin{abstract}
For the estimation of the intercalated hydrogen role in doping of the quasi-free-standing epitaxial graphene two systems are considered: (i) graphene- $\mathrm{N} H-\mathrm{SiC}\{0001\}$ and (ii) graphene-hydrogen monolayer- $\mathrm{N} H-\mathrm{SiC}\{0001\}$, where $\mathrm{N}=4,6$. In the (i) case Dirac point shift is due to the field of the substrate spontaneous polarization, in the (ii) case Dirac point shifts by the field of the electrical double layer formed by hydrogen adatoms. It is shown that in the first case (compared with the second one) hole concentration is increased and electron concentration is decreased. This results are in agreement with the experimental data available.
\end{abstract}

